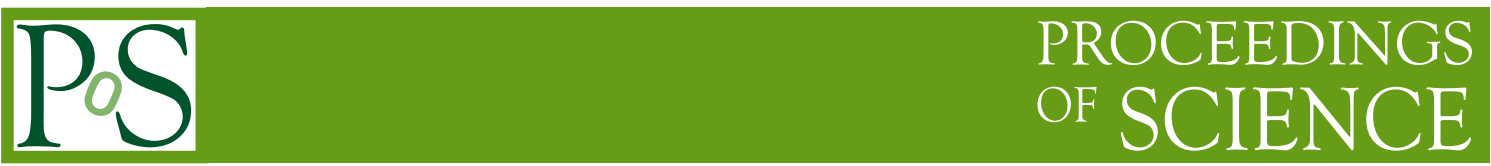

\title{
On the neutrino emission from BL Lacs
}

\author{
Maria Petropoulou* \\ Purdue University \\ E-mail: mpetropo@purdue.edu
}

\begin{abstract}
The recent IceCube discovery of $0.1-1 \mathrm{PeV}$ neutrinos of astrophysical origin opens up a new era for high-energy astrophysics. There are various astrophysical candidate sources, including active galactic nuclei (AGN) and starburst galaxies. Yet, a firm association of the detected neutrinos with one (or more) of them is still lacking. This talk will focus on the possible association of IceCube neutrinos with BL Lacs, a sub-class of radio loud AGN. We present the results from leptohadronic modeling of six individual BL Lacs, including the closest to Earth, Mrk 421, that were selected as probable counterparts of the IceCube neutrinos. We also show the cumulative neutrino emission from BL Lacs, which was calculated by incorporating our results from the modeling of individual sources into Monte Carlo simulations of the blazar population. We finally discuss our results in the light of current IceCube limits (above $2 \mathrm{PeV}$ ) and a possible future detection.
\end{abstract}

The 34th International Cosmic Ray Conference

30 July- 6 August, 2015

The Hague, The Netherlands

${ }^{*}$ Speaker. 


\section{Introduction}

The IceCube Neutrino Telescope based at the South Pole has detected a number of very highenergy neutrinos $(30 \mathrm{TeV}-2 \mathrm{PeV}$ ) of astrophysical origin during the first three years of data sampling in full configuration [1]. With a sample of 37 events the background only hypothesis with no astrophysical component has been rejected with a significance of more than $5 \sigma$.

If we exclude a possible connection with dark matter (e.g. [2]), the IceCube discovery calls for astrophysical sites, where cosmic-ray (CR) acceleration at energies close to and above the "knee" of the CR spectrum is efficiently at work. A firm association between the detected neutrinos and a specific class (or classes) of astrophysical sources is still lacking, notwithstanding the numerous scenarios that have been proposed so far. A pure Galactic origin of the observed neutrino signal is strongly constrained by diffuse TeV-PeV $\gamma$-ray limits (see e.g. [3] and references therein), but a Galactic contribution to the observed neutrino flux cannot be excluded at the moment. Protonproton $(p p)$ collisions in galaxy groups and/or star forming galaxies have also been invoked to explain the diffuse neutrino flux (e.g. [4]). Other extragalactic scenarios that include neutrino production through photohadronic $(p \gamma)$ interactions have been extensively discussed in the literature (see e.g. [5, 6, 7, 8, 9] for active galactic neuclei (AGN) scenarios and $[10,11,12]$ for gamma-ray burst (GRB) scenarios).

[13] have recently searched for plausible astrophysical counterparts within the median error circles of IceCube neutrinos using a phenomenological, yet model-independent method. This is based upon (i) the use of high-energy $\gamma$-ray $(\mathrm{GeV}-\mathrm{TeV})$ source catalogs, (ii) the assumption that each neutrino event is associated with one astrophysical source, and (iii) an energetic criterion, according to which a source is ruled out as a plausible counterpart, if the extrapolation of the $\gamma$-ray flux at the highest photon energies cannot explain the neutrino flux even after taking into account the large uncertainty of the latter. Using this method [13] derived the most probable counterparts for 9 out of the 18 neutrino events of their sample. Interestingly, these include 8 BL Lac objects, amongst which the nearest blazar, Mrk 421, and two pulsar wind nebulae. We note that flat spectrum radio quasars (FSRQs), which are believed to be more efficient PeV neutrino emitters than BL Lacs (e.g. [14]), do not appear in the list of most probable counterparts. This could be a consequence of the energetic criterion described above.

\section{The Model}

Motivated by the aforementioned results, we investigate the BL Lac-PeV neutrino connection within a specific theoretical framework for blazar emission [15], where the $\gamma$-rays are of photohadronic origin and the low-energy emission of the blazar SED is attributed to synchrotron radiation of primary relativistic electrons (the model and the numerical treatment are described, in more general terms, in [16] and [17]). Under the assumption that CR acceleration to energies $\sim 10-100 \mathrm{PeV}$ is viable in blazar jets, the production of charged pions is a natural outcome of photopion $(p \pi)$ interactions between the relativistic protons and the internally produced synchrotron photons. The decay of charged pions results in the injection of secondary relativistic electron-positron pairs. It is the synchrotron radiation of the latter that emerges in the $\mathrm{GeV}-\mathrm{TeV}$ regime, in contrast to the hadronic cascade scenario for blazar emission [18], where the cascade emission mainly contributes 


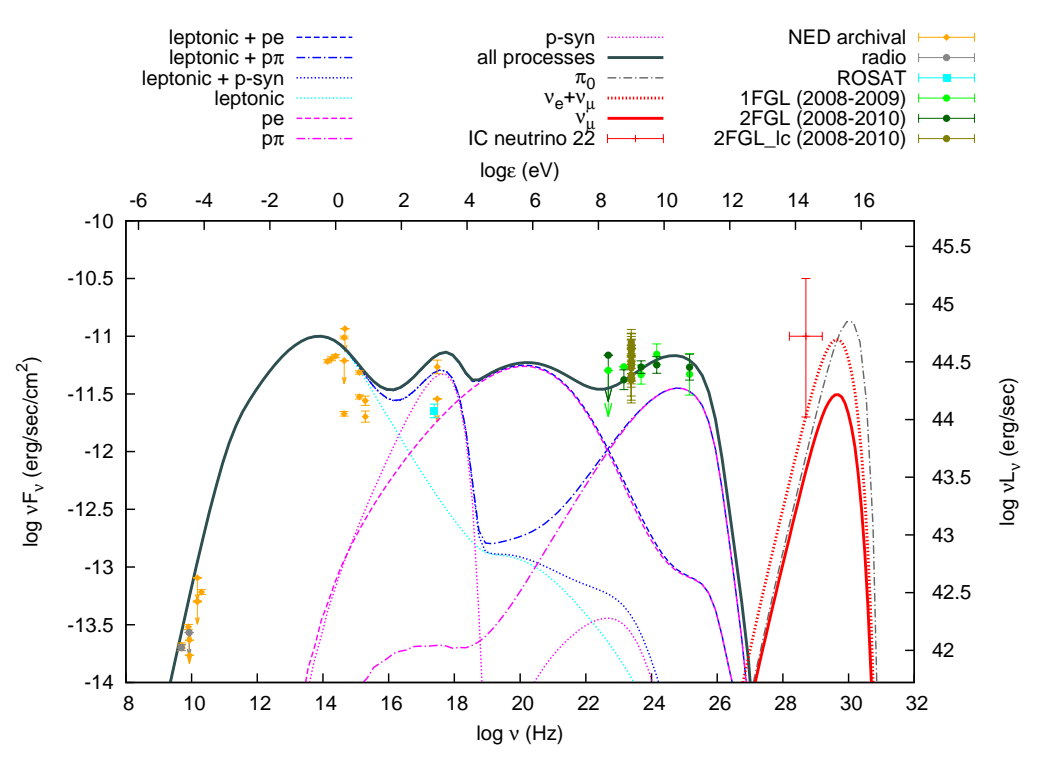

Figure 1: SED of blazar H 1914-194 and the neutrino flux for the corresponding IceCube event (ID 22). The data are obtained from ASI Science Data Center using the SED builder tool. All types of symbols and lines are explained in the legend above the plot.

to the $\gamma$-ray regime. As the synchrotron self-Compton (SSC) emission from primary electrons may also emerge in the $\mathrm{GeV}-\mathrm{TeV}$ energy band, the observed $\gamma$-ray emission can be totally or partially explained by photohadronic processes, depending on the specifics of individual sources [15]. An example of SED fitting with the leptohadronic model described above is shown in Fig. 1.

\section{Neutrino emission from individual BL Lacs}

The (quasi)-simultaneous SED of six BL Lacs, namely Mrk 421, 1ES 1011+496, PG 1553+113, H 2356-309, 1H 1914-194, and 1RXS J054357.3-553206, were fitted [15] with the leptohadronic model described in $\S 2$. The all-flavour neutrino fluxes derived by the model are presented in Fig. 2. According to the model-independent analysis of [13] the neutrino event 9 has two plausible astrophysical counterparts: the BL Lacs Mrk 421 and 1ES 1011+496. The differences between the neutrino fluxes originate from the differences in their SEDs. In this regard, the case of neutrino event 9 reveals in the best way how detailed information from the photon emission may be used to lift possible degeneracies between multiple astrophysical counterparts. As the neutrino spectrum for 1ES 1011+496 (dashed line) is an upper limit, our results strongly favour Mrk 421 against 1ES $1011+496$.

In all cases, the model-derived neutrino flux at the energy bin of the detected neutrino is below the $1 \sigma$ error bars, but still within the $3 \sigma$ error bars. Although the association of these sources cannot be, strictly speaking, excluded at the present time, blazars Mrk 421 and 1H 1914-194 are the two most interesting cases, because their association with the respective IceCube events can be either verified or disputed in the near future. Figure 2 demonstrates that the model-derived neutrino spectra from blazars with different properties are similar in shape. We may thus model the observed 


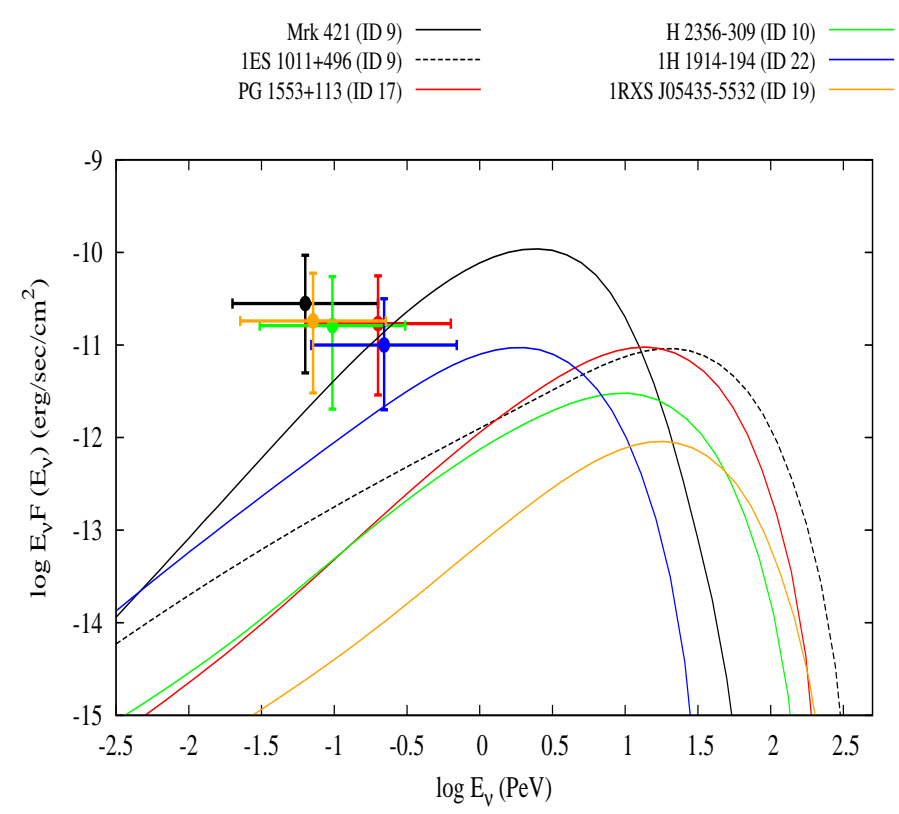

Figure 2: Comparison of the model (lines) and the observed (circles) neutrino fluxes as defined in [13] for the six BL Lacs of the sample. The Poissonian $1 \sigma$ error bars for each event are also shown [19].

differential neutrino plus anti-neutrino $(v+\bar{v})$ flux of all flavours $\left(F_{v}\left(E_{v}\right)\right)$ as

$$
F_{v}\left(E_{v}\right)=F_{0} E_{v}^{\beta} \exp \left(-\frac{E_{v}}{E_{0}}\right)
$$

where $\langle\beta\rangle \sim 0.34$ [15] and $E_{0}$ is in good approximation equal to the peak energy of the neutrino spectrum, namely

$$
E_{v, p}\left(\delta, z, v_{\mathrm{s}, \mathrm{p}}\right) \simeq \frac{17.5 \mathrm{PeV}}{(1+z)^{2}}\left(\frac{\delta}{10}\right)^{2}\left(\frac{v_{\mathrm{s}, \mathrm{p}}}{10^{16} \mathrm{~Hz}}\right)^{-1}
$$

In the above, $\delta$ is the Doppler factor, $z$ is the source redshift and $v_{\mathrm{s}, \mathrm{p}}$ is the observed synchrotron peak frequency. The luminosity from the $p \pi$ component is directly connected to that of very highenergy $(\sim 2-20 \mathrm{PeV})$ neutrinos (see e.g. Fig. 1). Thus, our approach allows us to associate the observed blazar $\gamma$-ray flux with the expected all-flavour neutrino flux as

$$
F_{v, \text { tot }}=Y_{v \gamma} F_{\gamma}\left(>E_{\gamma}\right),
$$

where $E_{\gamma}=10 \mathrm{GeV}$ and $Y_{v \gamma}$ is a factor that includes all the details about the efficiency of $p \pi$ interactions; for example, $Y_{v \gamma} \ll 1$ implies an SSC origin for the blazar $\gamma$-ray emission. The normalization $F_{0}$ can be then inferred from Eqs. (3.1) and (3.3).

\section{The neutrino background from BL Lacs}

The calculation of the neutrino background ${ }^{1}$ (NBG) from all BL Lacs requires detailed knowledge of the blazar population in terms of $v_{\mathrm{s}, \mathrm{p}}, \delta, \gamma$-ray fluxes and redshift. All these parameters,

\footnotetext{
${ }^{1}$ We use "background" in the astronomical sense of the total emission from a population. This includes resolved and
} 


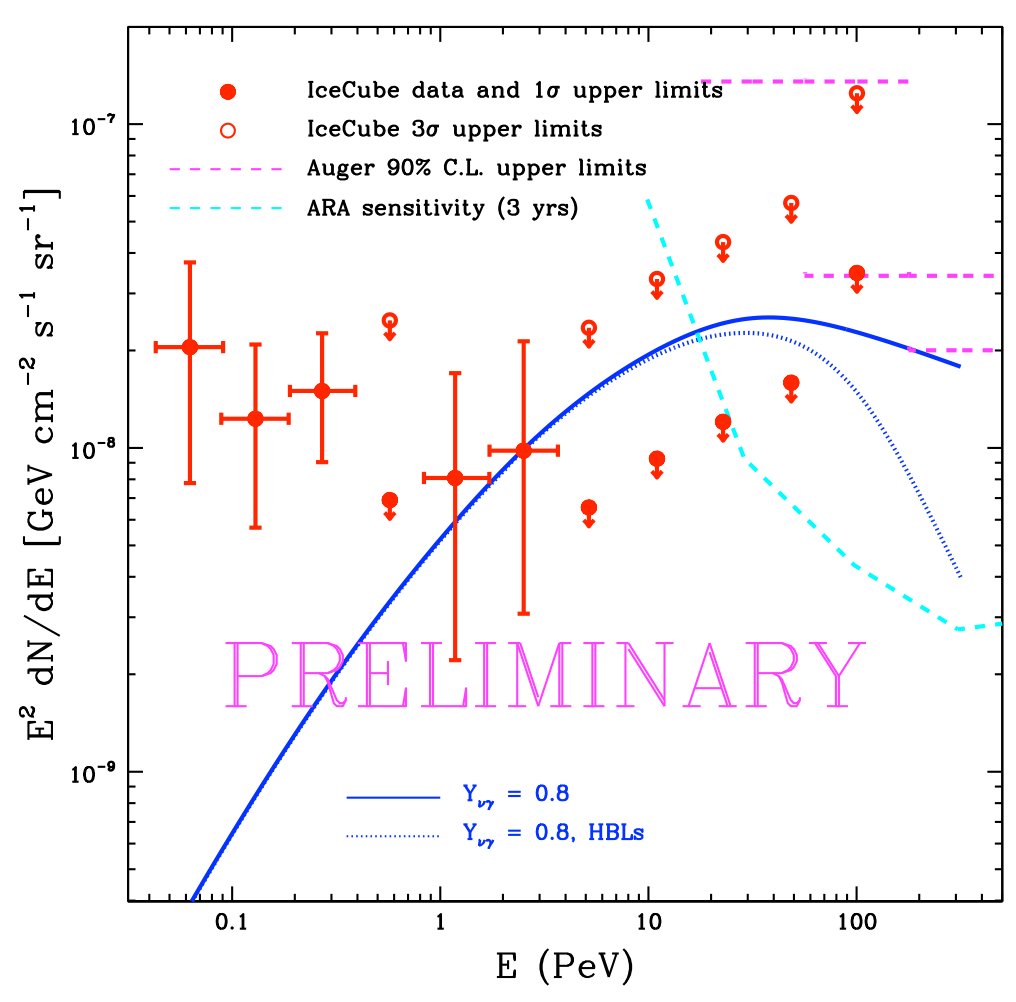

Figure 3: The predicted neutrino background (per neutrino flavour) from all BL Lacs (blue solid line) and from high-frequency peaked BL Lacs (HBL) only (blue dotted line) for $Y_{v \gamma}=0.8$ and $E_{\text {break }}=200 \mathrm{GeV}$, $\Delta \Gamma=0.5$. The curves correspond to the mean value of ten different simulations. The (red) filled points are the data points from [1], while the open points are the $3 \sigma$ upper limits. The upper (magenta) short dashed line represents the $90 \%$ C.L. upper limits from [23] while the lower (cyan) short dashed line is the expected three year sensitivity curve for the Askaryan Radio Array [24].

and many more, are available in the Monte Carlo simulations presented in a series of papers by Giommi, Padovani, et al. (e.g. [20], [21]). We note that the blazar SEDs in the simulations are extrapolated to the VHE band by using the simulated Fermi fluxes and spectral indices and assuming a break at $E=E_{\text {break }}$ and a steepening of the photon spectrum by $\Delta \Gamma$ (for details, see [22]). Using Eqs. (3.1)-(3.3) we assign to each blazar in the simulation a neutrino spectrum. Since $E_{0}$ is fully determined by Eq. (3.2) and $\beta$ covers a narrow range, we are left only with $Y_{v \gamma}$ as a possible "tunable" parameter. Then, we compute the total NBG as $B=\int_{F_{\min }}^{F_{\max }} F \frac{d N}{d F} d F$ where $\frac{d N}{d F}$ are the differential number counts and $F_{\min }$ and $F_{\max }$ are the fluxes over which these extend. To obtain the NBG per neutrino flavour we divide our results by three. Finally, we perform ten simulations and calculate their average in order to smooth out the "noise" inherent to the Monte Carlo simulations.

Preliminary results for the predicted NBG from BL Lacs are presented in Fig. 3. These are obtained for $Y_{v \gamma}=0.8, E_{\text {break }}=200 \mathrm{GeV}$, and $\Delta \Gamma=0.5$. We find that BL Lacs as a class (blue solid line) can easily explain the whole NBG at $E_{v} \gtrsim 0.5 \mathrm{PeV}$, while they do not contribute much

unresolved sources, the contribution of the latter corresponding to the "diffuse" intensity generally used by high-energy physicists. 
( $\sim 10 \%)$ at lower energies. At $E_{v} \lesssim 30 \mathrm{PeV}$ most of the contribution to the NBG comes from highfrequency peaked BL Lacs (HBL) (blue dotted line); see also Eq. (3.2). Although HBL represent a small fraction $(\sim 5 \%)$ of the BL Lac population, they dominate the neutrino output up to $\approx 30$ $\mathrm{PeV}$ due to their relatively high $\gamma$-ray, and therefore neutrino, fluxes and powers. According to preliminary calculations our results up to $\sim 1-2 \mathrm{PeV}$ are not sensitive on whether $Y_{v \gamma}$ is constant or dependent on the blazar $\gamma$-ray luminosity. However, assuming an anti-correlation between $Y_{v \gamma}$ and $L_{\gamma}(>10 \mathrm{GeV})$, we find that the predicted NBG at $E_{v} \gtrsim 5 \mathrm{PeV}$ is in tension with the $3 \sigma$ IceCube upper limits and the $90 \%$ C.L. upper limits from [23]. Thus, this hypothesis is ruled out.

The model prediction on the detectability of $2 \mathrm{PeV}<E_{v}<10 \mathrm{PeV}$ neutrinos for the NBG shown in Fig. 3 is $N_{v} \sim 4.6$ without taking into account the Glashow resonance (and $N_{v} \sim 7$, otherwise). This calculation is based on the effective areas from [25]. Since the model NBG peaks at $E_{v}>10 \mathrm{PeV}$, we expect 2-3 additional events up to $\sim 100 \mathrm{PeV}$ after making an educated guess on the effective areas above $10 \mathrm{PeV}$. Given that the $3 \sigma$ upper limit for 0 events is 6.6 [19], the prediction of $N_{v} \sim 6.6-7.6$ is close to being inconsistent with the IceCube non-detections. However, $Y_{v \gamma}=0.8$ is likely an upper limit. This was derived, in fact, from a small sample of BL Lacs, which may represent the tip of the iceberg in terms of neutrino emission, as they were selected as the most probable candidates [13]. For example, if $Y_{v \gamma}=0.3$ then we expect $N_{v} \approx 3$ (4) for $2<E_{v}<100 \mathrm{PeV}$, which is well within the $2 \sigma$ limit for 0 events.

\section{Summary and discussion}

Following the identification of eight BL Lac objects as likely sources of IceCube neutrinos by [13], we set out to deduce the neutrino emission of the ones whose redshift is known (6) by applying a leptohadronic model. We found that the model neutrino flux for two of them, namely

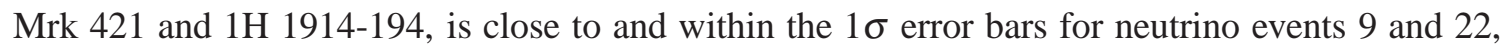
respectively.

The different model predictions for the neutrino emission from individual blazars originate from differences in their SEDs. In particular, we found that some cases favour fits dominated by SSC while others favour fits dominated by photohadronic interactions, with the former having a lower ratio of neutrino to $\gamma$-ray $\left(>10 \mathrm{GeV}\right.$ ) luminosities than the latter. This ratio, $Y_{v \gamma}$, stands out as an important parameter for the calculation of the NBG from BL Lacs.

We showed that a comparison of the model predicted NBG with current IceCube upper limits and, ultimately, future detections at $E_{v}>2 \mathrm{PeV}$, can be used to constrain the value of $Y_{v \gamma}$, since it is the only tunable parameter of the model. In other words, this would provide an indirect way of probing the origin of the BL Lac $\gamma$-ray emission.

A simultaneous look at the $\gamma$-ray (e.g. [21]) and neutrino backgrounds lead us to suggest that another population/physical mechanism could explain both the former at $E \lesssim 10 \mathrm{GeV}$ (since blazars dominate above that energy) and the latter at $E_{v} \lesssim 0.5 \mathrm{GeV}$. This might include star forming galaxies, although a Galactic component for the low-energy IceCube events could also be possible. Even if this is the case, we note that there is still room for individual BL Lac sources, like Mrk 421, to be sub-PeV neutrino emitters. 


\section{References}

[1] M. G. Aartsen, M. Ackermann, J. Adams, J. A. Aguilar, M. Ahlers, M. Ahrens, D. Altmann, T. Anderson, C. Arguelles, T. C. Arlen, and et al., Observation of High-Energy Astrophysical Neutrinos in Three Years of IceCube Data, Physical Review Letters 113 (Sept., 2014) 101101, [arXiv: 1405.5303$]$.

[2] J. F. Cherry, A. Friedland, and I. M. Shoemaker, Neutrino Portal Dark Matter: From Dwarf Galaxies to IceCube, ArXiv e-prints (Nov., 2014) [arXiv: 1411 .1071].

[3] M. Ahlers and K. Murase, Probing the Galactic origin of the IceCube excess with gamma rays, Physical Review D 90 (July, 2014) 023010, [arXiv:1309.4077].

[4] A. Loeb and E. Waxman, The cumulative background of high energy neutrinos from starburst galaxies, Journal of Cosmology and Astroparticle physics 5 (May, 2006) 3, [astro-ph/ 0601695 ].

[5] F. W. Stecker, C. Done, M. H. Salamon, and P. Sommers, High-energy neutrinos from active galactic nuclei, Physical Review Letters 66 (May, 1991) 2697-2700.

[6] F. Halzen and E. Zas, Neutrino Fluxes from Active Galaxies: A Model-Independent Estimate, Astrophysical Journal 488 (Oct., 1997) 669-674, [astro-ph / 9702193 ].

[7] J. P. Rachen and P. Mészáros, Photohadronic neutrinos from transients in astrophysical sources, Physical Review D 58 (Dec., 1998) 123005, [astro-ph/9802280].

[8] A. Atoyan and C. D. Dermer, High-Energy Neutrinos from Photomeson Processes in Blazars, Physical Review Letters 87 (Nov., 2001) 221102, [astro-ph / 0108053 ].

[9] A. Mücke, R. J. Protheroe, R. Engel, J. P. Rachen, and T. Stanev, BL Lac objects in the synchrotron proton blazar model, Astroparticle Physics 18 (Mar., 2003) 593-613, [astro-ph/ 0206164 ].

[10] E. Waxman and J. Bahcall, High Energy Neutrinos from Cosmological Gamma-Ray Burst Fireballs, Physical Review Letters 78 (Mar., 1997) 2292-2295, [astro-ph / 9701231 ].

[11] K. Murase, Prompt high-energy neutrinos from gamma-ray bursts in photospheric and synchrotron self-Compton scenarios, PhysRevD 78 (Nov., 2008) 101302, [arXiv: 0807 . 0919].

[12] M. Petropoulou, D. Giannios, and S. Dimitrakoudis, Implications of a PeV neutrino spectral cut-off in gamma-ray burst models, Monthly Notices of the Royal Astronomical Society 445 (Nov., 2014) 570-580, [arXiv:1405.2091].

[13] P. Padovani and E. Resconi, Are both BL Lacs and pulsar wind nebulae the astrophysical counterparts of Ice Cube neutrino events?, Monthly Notices of the Royal Astronomical Society 443 (Sept., 2014) 474-484, [arXiv:1406.0376].

[14] C. D. Dermer, K. Murase, and Y. Inoue, Photopion Production in Black-Hole Jets and Flat-Spectrum Radio Quasars as PeV Neutrino Sources, ArXiv e-prints (June, 2014) [arXiv: 1406.2633 ].

[15] M. Petropoulou, S. Dimitrakoudis, P. Padovani, A. Mastichiadis, and E. Resconi, Photohadronic origin of $\gamma$-ray BL Lac emission: implications for IceCube neutrinos, Monthly Notices of the Royal Astronomical Society 448 (Apr., 2015) 2412-2429, [arXiv : 1501 . 0711 ].

[16] S. Dimitrakoudis, A. Mastichiadis, R. J. Protheroe, and A. Reimer, The time-dependent one-zone hadronic model. First principles, Astronomy \& Astrophysics 546 (Oct., 2012) A120, [arXiv:1209.0413].

[17] S. Dimitrakoudis, M. Petropoulou, and A. Mastichiadis, Self-consistent neutrino and UHE cosmic ray spectra from Mrk 421, Astroparticle Physics 54 (Feb., 2014) 61-66, [arXiv: 1310 . 7923]. 
[18] K. Mannheim, P. L. Biermann, and W. M. Kruells, A novel mechanism for nonthermal X-ray emission, Astronomy \& Astrophysics 251 (Nov., 1991) 723-731.

[19] N. Gehrels, Confidence limits for small numbers of events in astrophysical data, Astrophysical Journal 303 (Apr., 1986) 336-346.

[20] P. Giommi, P. Padovani, G. Polenta, S. Turriziani, V. D’Elia, and S. Piranomonte, A simplified view of blazars: clearing the fog around long-standing selection effects, Monthly Notices of the Royal Astronomical Society 420 (Mar., 2012) 2899-2911, [arXiv: 1110 . 4706 ].

[21] P. Giommi and P. Padovani, A simplified view of blazars: contribution to the X-ray and $\gamma$-ray extragalactic backgrounds, Monthly Notices of the Royal Astronomical Society 450 (July, 2015) 2404-2409, [arXiv:1504.0197].

[22] P. Padovani and P. Giommi, A simplified view of blazars: the very high energy $\gamma$-ray vision, Monthly Notices of the Royal Astronomical Society 446 (Jan., 2015) L41-L45, [arXiv: 1410 . 0497 ].

[23] The Pierre Auger Collaboration, A. Aab, P. Abreu, M. Aglietta, M. Ahlers, E.-J. Ahn, I. Albuquerque, I. Allekotte, J. Allen, P. Allison, and et al., The Pierre Auger Observatory: Contributions to the 33rd International Cosmic Ray Conference (ICRC 2013), ArXiv e-prints (July, 2013) [arXiv:1307.5059].

[24] Ara Collaboration, P. Allison, J. Auffenberg, R. Bard, J. J. Beatty, D. Z. Besson, S. Böser, C. Chen, P. Chen, A. Connolly, J. Davies, M. Duvernois, B. Fox, P. W. Gorham, E. W. Grashorn, K. Hanson, J. Haugen, K. Helbing, B. Hill, K. D. Hoffman, E. Hong, M. Huang, M. H. A. Huang, A. Ishihara, A. Karle, D. Kennedy, H. Landsman, T. C. Liu, L. Macchiarulo, K. Mase, T. Meures, R. Meyhandan, C. Miki, R. Morse, M. Newcomb, R. J. Nichol, K. Ratzlaff, M. Richman, L. Ritter, C. Rott, B. Rotter, P. Sandstrom, D. Seckel, J. Touart, G. S. Varner, M.-Z. Wang, C. Weaver, A. Wendorff, S. Yoshida, and R. Young, Design and initial performance of the Askaryan Radio Array prototype EeV neutrino detector at the South Pole, Astroparticle Physics 35 (Feb., 2012) 457-477, [arXiv: 1105 . 2854 ].

[25] M. G. Aartsen, R. Abbasi, M. Ackermann, J. Adams, J. A. Aguilar, M. Ahlers, D. Altmann, C. Arguelles, J. Auffenberg, X. Bai, and et al., Probing the origin of cosmic rays with extremely high energy neutrinos using the IceCube Observatory, Physical Review D 88 (Dec., 2013) 112008.

\section{Acknowledgements}

MP: Support for this work was provided by NASA through Einstein Postdoctoral Fellowship grant number PF3 140113 awarded by the Chandra X-ray Center, which is operated by the Smithsonian Astrophysical Observatory for NASA under contract NAS8-03060. ER is supported by a Heisenberg Professorship of the Deutsche Forschungsgemeinschaft (DFG RE 2262/4-1). 